\title{
Quimioterapia da malária
}

\author{
um século no desenvolvimento de antimaláricos
}

\author{
NUNO VALE ${ }^{1}, \quad$ R I MOREIRA ${ }^{2}$ E PAULA GOMES ${ }^{1, *}$
}

A quimioterapia da malária é uma área de investigação que envolve esforços à escala mundial, devido à complexidade da interacção fármaco-parasita. A resistência aos fármacos surge em todas as regiões onde se manifesta o
Plasmodium e, neste artigo de revisão, pretende-se descrever o passado, presente e futuro de compostos antimaláricos que têm representado as esperanças e desilusões de um percurso longo e sinuoso.

\section{Introdução}

Até ao final do ano de 2004, 107 países ou territórios onde vivem aproximadamente 3200 milhões de pessoas, eram áreas de alto risco de transmissão da malária. A malária representa um grave problema, não só em termos de saúde pública, mas também ao nível do desenvolvimento cultural e sócio-económico das regiões tropicais e subtropicais do planeta. Segundo a OMS, registam-se anualmente cerca de 300 milhões de novos casos de malária aguda em todo o mundo, dos quais aproximadamente três milhões culminam na morte. Cerca de $60 \%$ dos novos casos de malária registam-se na África sub-sahariana, onde ocorrem $90 \%$ dos casos fatais de malária humana, $75 \%$ dos quais incidindo sobre crianças com idades inferiores a 5 anos. A malária é a principal causa de mortalidade infantil em África, sendo também responsável por cerca de $10 \%$ dos casos de morbilidade geral da população africana e consumindo cerca de $40 \%$ das despesas com Saúde Pública [1].

A malária é transmitida pela fêmea do mosquito Anopheles ao hospedeiro humano, e são quatro as espécies de pa-

\footnotetext{
${ }^{1}$ Centro de Investigação em Química da Universidade do Porto - Departamento de Química da Faculdade de Ciências do Porto

${ }^{2}$ Centro de Estudos de Ciências Farmacêuticas - Faculdade de Farmácia da Universidade de Lisboa

*pgomes@fc.up.pt
}

rasitas responsáveis pela doença (Plasmodium falciparum, $P$. vivax, $P$. malariae e $P$. ovale) sendo que, em termos de virulência e mortalidade, a espécie $P$. falciparum é a mais importante [2].

O ciclo de vida do Plasmodium (Figura 1) [3] inicia-se com a picada de um mosquito na pele humana, sendo os esporozoítos do parasita transferidos para a corrente sanguínea. Mais tarde, estes esporozoítos invadem as células do fígado, dando-se início à divisão assexuada do parasita, da qual resulta a produção de milhares de merozoítos. Estes merozoítos deixam as células hepáticas e vão infectar os eritrócitos da corrente sanguínea, onde tem início uma nova fase da reprodução assexuada, em ciclos de 48 horas. Através de estágios circulares, o parasita desenvolve-se em trofozoítos e esquizontes para, numa fase seguinte, cada esquizonte se dividir em 16 merozoítos eritrocíticos, que são libertados do eritrócito para invadir, logo de seguida, um novo eritrócito. Durante a fase eritrocítica, o parasita utiliza a hemoglobina do hospedeiro como fonte de aminoácidos para a sua reprodução.

Num processo induzido pelo "stress", uma pequena porção dos merozoítos sanguíneos sofre diferenciação em gametócitos feminino e masculino, que são transmissíveis ao mosquito quando este pica um indivíduo infectado. No interior do intestino do mosquito, os gametócitos femininos dividem-se em macrogâmetas e os masculinos em 4 a 8 microgâmetas flagelados, dando-se início à reprodução sexuada do parasita. Estes gâmetas femininos e masculinos fundem-se e formam zigotos, que se transformam em oocinetos móveis. Estes penetram a parede do intestino e saem do seu interior através da membrana externa, sob a forma de oocistos. A divisão assexuada dentro destes oocistos produz milhares de esporozoítos que serão libertados aquando da ruptura do oocisto, migrando de seguida para as glândulas salivares do mosquito, estando reunidas as condições para que se inicie um novo ciclo de transmissão ao hospedeiro humano [2].

A grande complexidade do ciclo de vida do parasita explica as enormes dificuldades que têm vindo a ser experimentadas, ao longo dos tempos, para o estabelecimento de uma terapia antimalárica eficaz e segura. 0 desenvolvimento de um fármaco antimalárico ideal pressupõe a existência de uma actividade antiparasitária óptima com um mínimo de efeitos adversos para o hospedeiro. No entanto, e como se pretende demonstrar neste artigo, os vários fármacos antimaláricos desenvolvidos e patenteados ao longo dos anos deram origem a ciclos de esperança seguidos de desilusão, face aos problemas que foram surgindo, em especial a crescente resistência por parte do parasita. 


\section{Breve resenha histórica da quimioterapia da malária}

Têm sido usadas substâncias com propriedades antimaláricas desde tempos imemoriais. Ainda antes da Era Cristã, os chineses já tratavam a malária com Ch'ang shan, uma preparação que consistia na raiz pulverizada da planta $\mathrm{Di}$ chroa febrifuga, cujo princípio activo é o alcalóide febrifugina. Antes da chegada dos europeus ao continente americano, os índios peruanos usavam a casca da quina para o tratamento da malária. Em 1677, a casca de quina foi incluída na Farmacopeia de Londres sob a designação Cortex peruano, sendo este o primeiro registo oficial, na Europa, sobre quimioterapia da malária [4]. Em 1820, os químicos franceses Pelletier e Caventou isolaram a substância activa da casca da quina, o alcalóide quinina [5] (1a, Figura 2), trabalho que foi reconhecido e premiado pelo Instituto Francês de Ciências [4].

Sete décadas depois da identificação da quinina, Paul Ehrlich utilizou azul de metileno (2, Figura 2) para curar dois pacientes infectados com malária, após ter observado que aquele composto era eficaz contra o parasita. Esta foi a primeira vez que se utilizou, em humanos, uma substância sintética com fins terapêuticos [2]. Hoje, sabe-se que o azul de metileno inibe a glutationa-reductase do $P$. falciparum, interferindo no processo de homeostasia redox [2,6].

Em 1925, investigadores alemães modificaram a estrutura do azul de metileno e sintetizaram a pamaquina ou plasmoquina (3, Figura 2), sendo este o primeiro fármaco capaz de prevenir recidivas da malária vivax [2]. Sete anos depois, em 1932, foi desenvolvida a mepacrina, também designada atebrina ou quinacrina (4, Figura 2), activa contra os estágios sanguíneos do P. falciparum. Estes dois fármacos foram usados extensivamente na II Grande Guerra Mundial.

Foi precisamente a partir da II Grande Guerra Mundial que surgiram os maiores avanços na pesquisa e desenvolvimento de novas substâncias activas por parte das principais potências económicas e militares da época (Alemanha, França e Estados Unidos da América). No

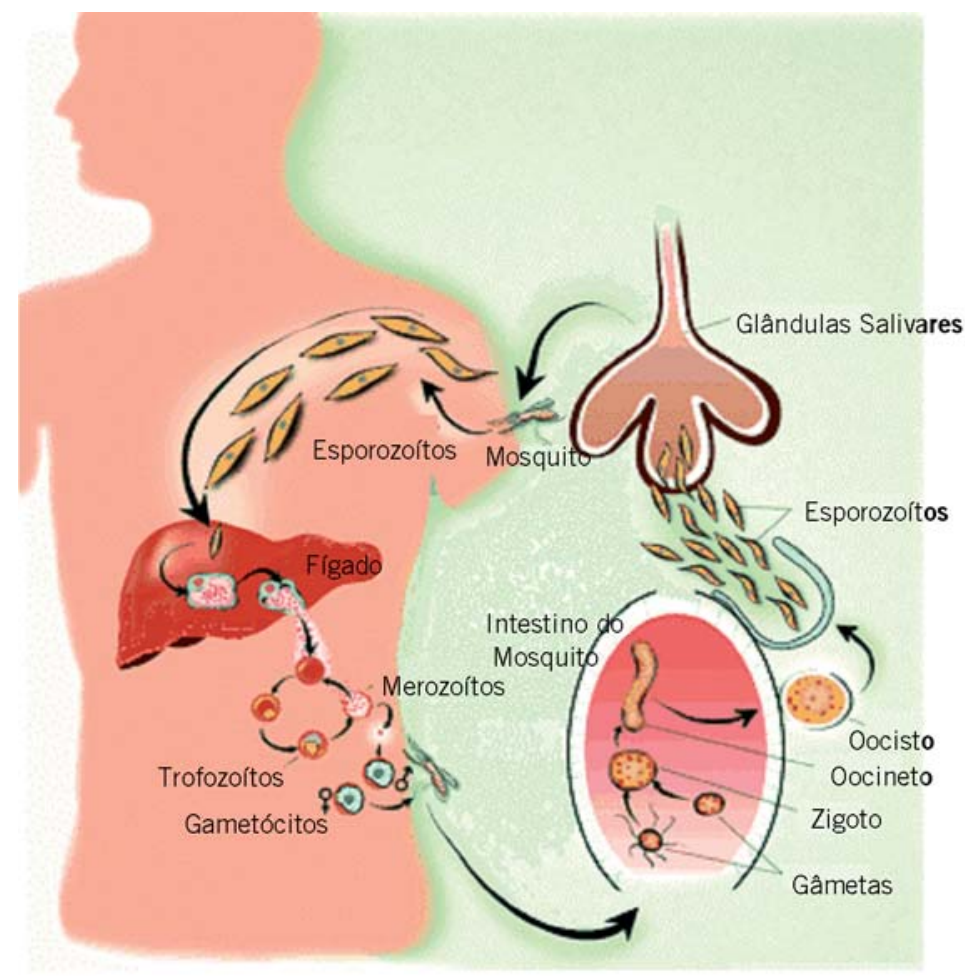

Figura 1 Ciclo de vida do parasita da malária humana (adaptado de [3])

âmbito de um programa para reposição dos "stocks" de quinina, que decorreu cinco anos antes do início da II Grande Guerra, foi concebida a cloroquina (CQ), o primeiro exemplo de 4-aminoquinolinas antimaláricas. Curiosamente, a CQ foi rapidamente abandonada em consequência da observação de efeitos tóxicos, numa triagem envolvendo quatro pacientes infectados com P. vivax. Em 1945, ensaios clínicos levados a cabo por investigadores americanos conduziram à recuperação da $C Q$ como fármaco de eleição no tratamento e profilaxia da malária. Contudo, o entusiasmo então criado em torno da aplicação da CQ como antimalárico deu lugar, na década de 60 , aos primeiros sinais de fracasso, quando se detectaram vários casos de resistência ao fármaco na América do Sul, Ásia e África. Tal facto conduziu ao relançamento, à escala mundial, de programas de pesquisa centrados na síntese de novos fármacos antimaláricos $[5,7]$.

A par da CQ surgiu, também em 1945, uma outra 4-aminoquinolina antimalári$\mathrm{ca}$, a amodiaquina (AQ) que, comparativamente à $C Q$, se apresenta mais activa contra $P$. falciparum e menos tóxica para os pacientes. Por estes motivos, a $A Q$ fez ressurgir a esperança para a cura da malária em zonas onde já havia sido detectada resistência à $C Q$, sendo largamente utilizada ao longo de quarenta anos, até ter sido associada a efeitos graves de agranulocitose e hepatotoxicidade, em $1986[5,8]$.

A primaquina $(P Q)$ foi sintetizada pela primeira vez nos Estados Unidos da América, em 1946, sendo o exemplo mais representativo das 8-aminoquinolinas antimaláricas, descritas em detaIhe mais adiante, e nas quais também se inclui o seu análogo pamaquina (3). A PQ é activa contra todas as formas exoeritrocíticas do parasita, incluindo os gametócitos. Assim, a PQ é o único fármaco antimalárico capaz de bloquear a transmissão da doença entre o hospedeiro humano e o mosquito, com evidentes vantagens para o controlo da propagação da malária. Contudo, a PQ é tóxica para pacientes com deficiência em glucose-6-fosfato (G6PD) eritrocitária, condição que provoca anemia hemolítica e confere maior resistência ao parasita [9-11]. O uso da PQ está igualmente limitado pelo facto da maioria das 8-aminoquinolinas causarem metemo- 
globinémia, ou seja, conversão da oxihemoglobina a metemoglobina, sendo esta incapaz de transportar o oxigénio aos diferentes tecidos [12,13].

Ainda durante a II Grande Guerra Mundial, investigadores ingleses desenvolveram o proguanil, que levou à síntese da pirimetamina em 1950 [2]. A descoberta da di-hidrofolato reductase (DHFR), enzima envolvida na síntese das bases pirimidínicas do ADN, como alvo do proguanil e pirimetamina permitiu associar estes fármacos com sulfonamidas inibidoras da di-hidropteroato sintetase (DHPS), também envolvida na síntese do ADN. O uso terapêutico do proguanil é limitado pelo desenvolvimento de resistência por parte das formas eritrocíticas do $P$. falciparum, mas continua a ser usado na profilaxia da malária em combinação com a CQ [14].

Na década de 60 foi sintetizado, na China e na França, o composto antimalárico bisquinolínico piperaquina (5, Figura 3), o qual foi largamente utilizado no tratamento e profilaxia da doença, na China e na Indonésia, ao longo das duas décadas seguintes. A sua eficácia era tão elevada, que passou a ser recomendado pelo Programa de Controlo da Malária Chinês, em 1978. No entanto, na década de 80 era já significativo o desenvolvimento de resistência ao fármaco, o que, aliado ao surgimento de novos fármacos promissores, fez diminuir a utilização da piperaquina. Dez anos mais tarde, a piperaquina foi recuperada, novamente na China, para aplicação em terapias de combinação com antimaláricos sesquisterpénicos, com recentes ensaios clínicos animadores [15].

Ainda nos anos 60 foi desenvolvida pelo Walter Reed Army Institute of Research (WRAIR) um novo antimalárico denominado halofantrina (6, Figura 3). O seu mecanismo de acção poderá ser similar ao da $C Q$ e da quinina, por formação de complexos tóxicos com protoporfirina férrica IX, provocando alterações na membrana do parasita $[5,14]$.

Durante os anos 70, em plena Guerra do Vietname, o Departamento de Defesa Norte-Americano, também através do WRAIR, concebeu a mefloquina ( $\mathbf{7}$, Figura 3), um derivado da quinina, para dar resposta à resistência do parasita aos fármacos existentes na época. A mefloquina tem sido usada nos últimos vinte anos contra estirpes do parasita resistentes à $\mathrm{CQ}$, mas o uso deste fármaco tem sido associado a efeitos neuropsiquiátricos adversos, nomeadamente, ansiedade, depressão e alucinações [15a].

A amopiroquina (8, Figura 3), conhecida desde 1957, é uma 4-aminoquinolina estruturalmente semelhante à $A Q$ que tem sido eficaz no tratamento de $P$. falciparum e $P$. vivax ao longo dos últimos quarenta anos. Sofreu, contudo, um relativo abandono em detrimento da CQ com o decorrer dos ensaios clínicos, tendo sido recuperada nos anos 80 e 90, devido ao aparecimento de resistência do parasita à $C Q$ e aos bons resultados a que conduz após profilaxia prolongada [16].

A lumefantrina, ou benflumetol (9, Figura 3), foi inicialmente sintetizada na Academia Militar das Ciências Médicas de Beijing, na China, há aproximadamente vinte anos. Neste momento, não existem dados clínicos da sua eficácia<smiles>[2H][C@@]1(C=C)C[C@@H]2CC[C@H](C1)N2[C@H](O)[C@H](O)c1ccnc2ccc(OC)cc12</smiles><smiles>CCN(CC)CCCC(C)Nc1cc(OC)cc2cccnc12</smiles>

Pamaquina<smiles>CCN(CC)CCCC(C)Nc1c2ccccc2nc2cc(Cl)ccc12</smiles>

Figura 2 Fármacos antimaláricos historicamente relevantes 
como monoterapêutico, mas existem estudos farmacocinéticos e farmacodinâmicos da sua acção em combinação com outros antimaláricos, nomeadamente, com o artemeter. Estes estudos permitiram verificar que, além da redução da extensão da parasitémia, a combinação daqueles dois fármacos previne o desenvolvimento de resistência a qualquer deles, em contraste com o que sucedia quando usados separadamente [17].

Em 1971 foi desenvolvido e registado na China um composto estruturalmente similar à mepacrina (4), CQ (10, Figura 4) e AQ (11, Figura 4), conhecido por pironaridina ou malaridina (12, Figura 4), apresentando actividade contra estirpes resistentes à CQ [2].

Em 1972 foi isolado o sesquisterpeno artemisinina, que deu origem a uma família de compostos antimaláricos que serão abordados em detaIhe mais adiante. A artemisinina provém da planta Artemisia annua, que já era usada na China há cerca de 2000 anos, para o tratamento de febres. A artemisinina é, fundamentalmente, um esquizonticida sanguíneo com uma acção muito rápida e mais potente que a da $C Q$ ou da quinina. Desde o seu isolamento e caracterização como antimalárico eficaz, a artemisinina foi a base para a concepção de vários derivados artemisínicos caracterizados por rápida absorção oral e boa distribuição tecidular [2,14].

Mais recentemente, o WRAIR desenvolveu a tafenoquina, que é uma 8-aminoquinolina concebida com o propósito de melhorar a eficácia e prolongar o efeito da primaquina. A tafenoquina é activa contra as formas hepáticas da malária, apresentando cura radical em macacos rhesus. É também eficaz contra as formas sanguíneas do parasita, apresentando uma actividade quatro a cem vezes superior à da primaquina em malária falciparum. Neste momento, a tafenoquina é um dos produtos investigacionais da multinacional farmacêutica GlaxoSmithKline, onde já decorrem ensaios clínicos de Fase III, e é usada no tratamento e profilaxia da malá-<smiles>Clc1ccc2c(N3CCN(CCCN4CCN(c5ccnc6cc(Cl)ccc56)CC4)CC3)ccnc2c1</smiles><smiles>CCCCN(CCCC)CC[C@H](O)c1cc(C(F)(F)F)cc2c1ccc1c(Cl)cc(Cl)cc12</smiles><smiles>O[C@H](c1cc(C(F)(F)F)nc2c(C(F)(F)F)cccc12)C1CCCCN1</smilesMefloquina<smiles>CCCCN(CCCC)C[C@H](O)c1cc(Cl)cc2c1-c1ccc(Cl)cc1/C2=C\c1ccc(Cl)cc1</smiles>

Figura 3 Alguns fármacos antimaláricos do pós-guerra

ria em soldados Norte-Americanos $[18,19]$.

A elubaquina, ou bulaquina, foi sintetizada no fim dos anos 80 , sendo a 8-aminoquinolina antimalárica mais recente. Demonstrou actividade contra recidivas da doença causada pela estirpe P. cynomolgi em macacos rhesus. Comparativamente à primaquina, a elubaquina é menos tóxica, com uma incidência de metemoglobinémia em cães cerca de três a quatro vezes inferior à registada para a primaquina. Em humanos, os valores obtidos com voluntários para ensaios clínicos da Fase I indicam que a elubaquina não induz a um aumento de metemoglobinémia [20].

\section{Fármacos antimaláricos mais representativos}

Como já se pode entrever na secção anterior, os fármacos antimaláricos englobam um grande número de moléculas orgânicas que são tradicionalmente distribuídas por três famílias principais:

a) amino-álcoois, que incluem os alcalóides da quina (Cinchona sp.), dos quais a quinina é o exemplo clássico, e todas as aminoquinolinas sintéticas;

b) derivados artemisínicos que, como o nome indica, englobam o sesquisterpeno artemisinina e seus derivados; 
c) antifolatos e outros, onde se incluem também antibióticos com actividade antimalárica, como biguanidas e sulfonamidas, e outros que não se encaixam nas duas famílias anteriores.

Os compostos mais representativos para o tratamento ou profilaxia da malária, como monoterapêuticos ou em combinação com outros fármacos, serão abordados com maior detalhe nesta secção.

\section{Alcalóides da quina}

A quinina (1a, Figura 2) e a quinidina (1b, Figura 2), dois dos quatro alcalóides extraídos da casca da quina (os outros são cinchonidina e cinchonina), são normalmente usadas como monoterapêuticos em pacientes com malária. No entanto, os seus efeitos adversos, quando em doses elevadas, e a crescente resistência do parasita aos alcalóides da quina, têm tornado necessária a combinação com um segundo fármaco.

\section{Quinina}

A quinina (1a) é rapidamente absorvida por via oral e tem razoável distribuição tecidular, mas apresenta baixo poder de acumulação, uma vez que é rapidamente metabolizada e excretada. Do metabolismo da quinina foram identificados quatro metabolitos no plasma e na urina, tendo-se determinado, por cromatografia líquida de alta eficiência (HPLC), que a 3-hidroxiquinina é o metabolito maioritário 48 horas após a administração de uma dose única de 500 mg de cloridrato de quinina [21]. A grande vantagem da quinina como antimalárico reside na sua solubilidade adequada para formulações intravenosas. Assim, assume particular relevância no tratamento de pacientes que não toleram a medicação oral, mas a elevada dose requerida na administração por via intravenosa pode causar arritmia cardíaca grave e mesmo hipotensão fatal [2,7].

\section{Quinidina}

A quinidina (1b) é o diastereoisómero da quinina e tem propriedades farmacocinéticas comparáveis. No entanto, apresenta menor afinidade para com as proteínas plasmáticas, pelo que pode atingir níveis de concentração e de distribuição tecidular superiores aos da quinina [14]. Em algumas regiões do planeta, como na Libéria e no Gabão, a quinidina é um antimalárico bastante eficaz, mas o seu uso está limitado devido aos efeitos adversos que provoca ao nível do sistema cardiovascular [22]. Por outro lado, um estudo permitiu constatar que a quinidina é mais eficaz do que a quinina contra estirpes brasileiras de P. falciparum, mas que tal situação se inverte no caso de algumas estirpes africanas, nomeadamente, em estirpes que circulam em Angola e no Uganda [22,23].

\section{Halofantrina}

A halofantrina $(\mathrm{HF}, 6)$ é efectiva contra estirpes de $P$. falciparum resistentes aos antimaláricos clássicos, em algumas regiões do mundo. Este fármaco quiral é administrado como mistura racémica, e a concentração do enantiómero (+) no plasma humano é mais alta do que o enantiómero (-), resultados estes confirmados por estudos in vitro.[24] A estereoespecificidade observada na ligação da HF às proteínas plasmáticas repercute-se sobre o potencial cardiotóxico do fármaco, que diminui por redução da proporção de (+)-HF. O carbono estereogénico da (-)-HF tem a mesma configuração do carbono correspondente no isómero $S$ da quinina, ao passo que o enantiómero (+)-HF tem idêntica correspondência em relação à quinidina. Estes dados apoiam a existência de estereoespecificidade na cardiotoxicidade da HF [25].

A biotransformação da HF nos humanos conduz à perda dos grupos butilo ligados ao azoto, originando uma estrutura vulgarmente designada por "desbutil-halofantrina” (DHF), cuja farmacocinética em ratos evidencia estereosselectividade: a (+)-DHF é eliminada mais lentamente e distribuída menos extensamente que a (-)-DHF [26]. O facto da ( \pm )-HF ser um antimalárico eficaz apenas em concentrações elevadas, com sérios riscos de cardiotoxicidade, faz com que o desenvolvimento de derivados ou análogos da (-)-HF ou da (-)-DHF seja um dos rumos que está a ser seguido para a obtenção de antimaláricos mais seguros [27].

\section{Mefloquina}

A mefloquina (MFQ, 7) é um derivado quinolino-metanólico com dois centros quirais. Este fármaco é administrado clinicamente como uma mistura racémica dos enantiómeros (-)-(11S, 2'R)- e $(+)-\left(11 R, 2^{\prime} S\right)$-eritro. Contrariamente ao observado para outros antimaláricas quirais, a MFQ apresenta esterosselectividade in vitro a favor do enantiómero $(+)$, com uma razão eudísmica de 2:1 [22]. O modo de acção contra o P. falciparum é desconhecido, mas os seus efeitos estão confinados às fases sanguíneas do ciclo de vida do parasita. Este fármaco é extensamente absorvido pelo intestino, alcançando o pico de concentração plasmática duas horas após administração. É caracterizado por elevada lipofilicidade e grande volume aparente de distribuição. Apresenta um tempo de semivida de vinte dias, sendo eliminado essencialmente na forma de ácido carboxílico [28]. Porém, uma das desvantagens da MFQ é a elevada variabilidade demonstrada para as suas propriedades farmacocinéticas que podem conduzir a níveis subterapêuticos [14]. No entanto, e apesar de recentes relatos de efeitos adversos da MFQ, nomeadamente neurotoxicidade, esta continua a ser o único fármaco licenciado no Japão para a quimioprofilaxia da malária [29].

Vários estudos têm sido desenvolvidos nos últimos anos no sentido da aplicação da MFQ em combinação com outros fármacos, essencialmente derivados da artemisinina. Em alguns casos, estas combinações apresentam maior eficácia do que abordagens monoterapêuticas clássicas [30-33].

\section{Lumefantrina}

A lumefantrina (LF, 9) é um fármaco antimalárico pouco estudado, do ponto de vista farmacocinético. É caracterizada por uma baixa absorção e por ter uma biodisponibilidade oral reduzida e muito variável, dada a sua elevada lipofilicidade [17, 34, 35].

A nível terapêutico, a LF é combinada com um derivado artemisínico, o artemeter, sendo esta combinação comercializada sob a designação de "co-artemeter" [36, 37]. 
In vitro, a LF é parcialmente metabolizada a "desbutil-lumefantrina" (DLF), à semelhança do que ocorre com a HF. Não existem ainda dados conclusivos sobre a formação de DLF in vivo, e só recentemente foi validado um método bioanalítico para determinação de LF e DLF no plasma [38].

\section{4-Aminoquinolinas}

A investigação em antimaláricos 4-aminoquinolínicos reveste-se da maior importância, já que a CQ tem sido, apesar das desvantagens, o fármaco de maior eficácia global no tratamento e profilaxia da malária. Por outro lado, a síntese de 4-aminoquinolinas como a $C Q$ ou a $A Q$ é fácil e barata, e estes compostos são, de uma forma geral, bem tolerados e com níveis de toxicidade aceitáveis para o tratamento da malária aguda. Adicionalmente, a $A Q$ é eficaz contra determinadas estirpes do parasita resistentes à $C Q$. Este último aspecto justifica o recente interesse no desenvolvimento de novos derivados da $A Q$, com o intuito de conceber novos antimaláricos mais vantajosos.

\section{Cloroquina}

A cloroquina $(C Q, 10)$ é um dos quimioterápicos mais importantes da história da indústria Químico-Farmacêutica. A $C Q$ é um potente esquizonticida sanguí-<smiles>CCN(CC)CCC[C@H](C)Nc1ccnc2cc(Cl)ccc12</smiles>

10<smiles>COc1ccc2nc3cc(Cl)ccc3c(Nc3cc(CN4CCCC4)c(O)c(CN4CCCC4)c3)c2n1</smiles><smiles>CCN(CC)Cc1cc(Nc2ccnc3cc(Cl)ccc23)ccc1O</smiles>

Figura 4 Antimaláricos 4-aminoquinolínicos usados em ensaios clínicos

neo, eficaz contra as formas eritrocíticas de todas as espécies de Plasmodia. No entanto, não é activa contra esporozoítos ou hipnozoítos hepáticos, uma vez que o alvo terapêutico das 4-aminoquinolinas é o processo de destoxificação do grupo heme resultante da degradação da hemoglobina do hospedeiro [39].
O metabolismo da CQ tem vindo a ser estudado desde 1966, e inicia-se com a perda de um grupo $N$-etilo, originando "desetil-cloroquina" (DCQ) (13, Figura 5), que sofre transformações adicionais conducentes aos restantes metabolitos, como ilustrado na Figura 5 [40].<smiles>CCN(CC)CCCC(C)Nc1ccnc2cc(Cl)ccc12</smiles>

${ }_{\mathrm{CQ}}^{10}$<smiles>CC(CCC=O)Nc1ccnc2cc(Cl)ccc12</smiles>

15<smiles>CCNCCCC(C)Nc1ccnc2cc(Cl)ccc12</smiles>

13
$D C Q$<smiles>CC(CCC(=O)O)Nc1ccnc2cc(Cl)ccc12</smiles>

16<smiles>CC(CCCN)Nc1ccnc2cc(Cl)ccc12</smiles>

Figura 5 Degradação metabólica da CQ in vivo [39] 
Apesar dos vários metabolitos originados pela $C Q$, apenas uma pequena fracção é excretada na forma de ácido carboxílico (16) nos humanos, tendo a amina 17 uma importância bastante reduzida. 0 principal metabolito excretado é a DCQ, que apresenta actividade antimalárica semelhante à $\mathrm{CQ}[39,41]$.

A resistência do parasita à $C Q$ detectou-se pela primeira vez em 1959 na Ásia e América do Sul e veio impulsionar a busca de quimioterápicos antimaláricos alternativos [39,42]. A CQ é uma base fraca que se acumula nos vacúolos digestivos do parasita ( $\mathrm{pH}=5,0)$, em virtude do gradiente de $\mathrm{pH}$ entre este compartimento e o meio extracelular. Assim, um dos mecanismos de resistência do parasita poderá passar pela alteração do $\mathrm{pH}$ destes compartimentos, reduzindo-se os níveis de fármaco aí acumulados. Mas o principal mecanismo de resistência à $C Q$ envolve o transporte activo mediado por transportadores para o exterior da célula do parasita, de um modo análogo ao que acontece no fenómeno de resistência aos agentes antitumorais $[39,42]$.

A eficácia da $C Q$ pode ser melhorada através da sua combinação com outros antimaláricos, em especial com antifolatos [14].

\section{Amodiaquina}

A amodiaquina $(A Q, 11)$ distingue-se estruturalmente da $C Q$ por possuir o grupo 4-hidroxi-anilino ligado ao anel quinolínico. No entanto, ambas as 4-aminoquinolinas apresentam uma geometria global similar, com um espaçamento de quatro carbonos entre os grupos amino secundário e terciário. Koh e colaboradores sugeriram que a $A Q$ adopta uma "conformação bioactiva", na qual os azotos quinolínico e dialquilamínico estão separados de 8,30 A, comparável com a distância de 8,38 Å atribuída à $C Q$ [39]. No caso da $A Q$, tal "conformação bioactiva" só terá lugar se existir uma ligação de hidrogénio entre o grupo hidroxilo e o protão do grupo N,N-dialquilamónio (Figura 6).

A estrutura da base de Mannich é crucial para a actividade antimalárica da $A Q$ que, à semelhança da $C Q$, é um potente esquizonticida sanguíneo, eficaz mesmo contra alguns tipos de Plamodium resistentes à $\mathrm{CQ}$.

Recentemente, a $A Q$ foi reclassificada como pró-fármaco, pois é extensamente metabolizada ao metabolito activo "desetil-amodiaquina" (DAQ), por perda de um grupo $\mathrm{N}$-etilo, e ao metabolito secundário 2-hidroxi-amodiaquina, sendo ambos eliminados por excreção renal [14].

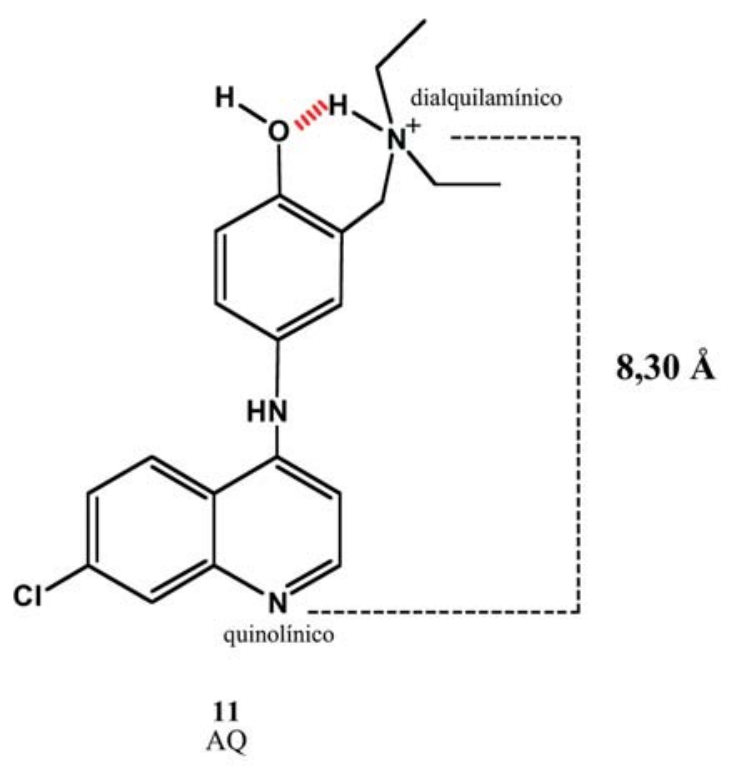

Em contraste com outros antimaláricos, a $A Q$ acumulada nos neutrófilos humanos dá origem a um metabolito electrofílico que contribui para a diminuição dos níveis de glutationa, resultando, portanto, numa toxicidade acrescida [43].

A redução da toxicidade da $A Q$ tem sido abordada nos últimos anos, através da obtenção de novos derivados da AQ. Um caso exemplar é a amidometilação da $A Q$ pela reacção desta com $N$-clorometilamidas, formando-se uma base de Mannich estável, caracterizada por uma excelente actividade in vitro contra $P$. falciparum [44].

Uma outra abordagem, bastante recente, baseia-se na combinação da $A Q$, ou do seu metabolito activo DAQ, com outros agentes antimaláricos, como a artemisinina ou antifolatos [45-47].

Pironaridina

A pironaridina (12) está integrada na classe das 4-aminoquinolinas antimaláricas, sendo um análogo da $A Q$ em que o anel quinolínico foi substituído por um núcleo de acridina. Apesar da sua farmacocinética ainda não estar bem esclarecida, a pironaridina é usada na China em combinação com outros fármacos, como a artimisinina, [14,47] e é considerada promissora para o tratamento de malária falciparum e vivax em zonas com forte resistência à $\mathrm{CQ}$, como África [39]. A pironaridina actua rapidamente e é bem tolerada em pacientes com malária aguda ou moderada, segundo parâmetros revelados no primeiro estudo efectuado fora da Ásia, em 1996 [48].

Estudos posteriores permitiram determinar que a pironaridina é um esquizonticida eficaz contra estirpes letais e multirresistentes de malária em roedores [49]. Por outro lado, Petmitr e colaboradores determinaram que a pironaridina possui uma actividade gametocitocida cerca de cem vezes superior à da $\mathrm{PQ}$, sendo esta última, actualmente, o único agente gametocitocida de uso clínico. Assim, a pironaridina poderá vir a ser um fármaco de eleição no combate à malária, pelo seu duplo efeito esquizonticida e gametocitocida $[50,51]$.

Figura 6 "Conformação bioactiva" proposta por Koh e colaboradores para a AQ [39] 


\section{8-Aminoquinolinas}

As 8-aminoquinolinas antimaláricas representam os clássicos agentes esquizonticidas teciduais, em contraposição com os esquizonticidas sanguíneos, sendo a primaquina (18, Figura 7) o membro mais importante da família, dada a sua potente actividade contra as formas teciduais primária e secundária do Plasmodium. Recentemente, modificações moleculares da $P Q$ conduziram a novas estruturas 8-aminoquinolínicas que apresentam actividade esquizonticida sanguínea. As novas potencialidades das 8-aminoquinolinas, tradicionalmente consideradas apenas pelo seu carácter gametocitocida, abrem novos horizontes para a quimioterapia da malária [52].

Primaquina

A PQ tem tido um papel crucial no combate à malária ao longo dos últimos 40 anos, uma vez que é o único agente que actua eficazmente contra todas as formas exoeritrocíticas do parasita. Dispondo de actividade contra os gametócitos de todas as espécies do parasita que causam a malária humana, a $P Q$ é o único antimalárico bloqueador da transmissão da doença [13]. No entanto, tal como já foi referido, a $\mathrm{PQ}$ não é eficaz em indivíduos com deficiência em G6PD, estando associada a efeitos adversos como anemia hemolítica ou metemoglobinémia. Após administração oral, a PQ é degradada metabolicamente por perda do grupo $\mathrm{N}$-alquilo, originando 6-metoxi-8-aminoquinolina (6-MAQ). Acredita-se que a 6-MAQ possa ser convertida no derivado $\mathrm{N}$-hidroxilado (MAQ-NOH, 6-metoxi8-hidroxiaminoquinolina), sendo este<smiles>COc1cc(NC(C)CCCN)c2ncccc2c1</smiles><smiles>COc1ccc2c(Oc3cccc(C(F)(F)F)c3)c(OC)cc(NC(C)CCCN)c2n1</smiles>

19<smiles>[2H]C(CCCNC1OCCC1C(C)=O)Nc1cc(OC)cc2cccnc12</smiles>

Tafenoquina

Figura 7 8-aminoquinolinas usadas na quimioterapia da malária

o agente responsável pela metemoglobinémia. Jollow e colaboradores [13] comprovaram a formação de MAQ-NOH nos microssomas hepáticos do rato e do homem, observando que a MAQ-NOH é um activador directo da metemoglobina. De facto, as 8-aminoquinolinas induzem a conversão da oxi-hemoglobina a metemoglobina, sendo esta incapaz de transportar o oxigénio aos diferentes tecidos [53].

A toxicidade da $P Q$ é directamente proporcional ao tipo e quantidade de metabolitos que origina, incluindo ainda a carboxiprimaquina $(\mathrm{PQ}-\mathrm{CX})$, formada por desaminação oxidativa da $\mathrm{PQ}$, dando seguidamente origem à correspondente amida e a derivados $\mathrm{N}$-acetilados. Estes, por seu turno, podem ser convertidos aos correspondentes compostos diméricos (em baixa percentagem) [54] e ao metabolito fenólico 5-hidroxiprimaquina (5-OH-PQ). Este metabolito gera espécies radicalares de oxigénio, acentuando a hematotoxicidade do fármaco parental [55].

Uma estratégia para melhorar a acção terapêutica da $P Q$, desenvolvida por nós [56-58] nos últimos anos, baseia-se na preparação de derivados $N$-acil-PQ (21, Figura 8), que são posteriormente convertidos em imidazolin-4-onas (22, Figura 8). A $N$-acilação da $P Q$, usando aminoácidos como agentes acilantes,<smiles>[Z]C(CCCN)Nc1cc(OC)cc2cccnc12</smiles>

Figura 8 Modificação da PQ para obtenção de imidazolidin-4-onas antimaláricas 
protege o fármaco parental face ao metabolismo oxidativo, enquanto que a posterior ciclização à imidazolidin-4-ona resulta numa estrutura resistente à hidrólise catalisada por aminopeptidases. Deste modo, pretende-se obter uma família de compostos antimaláricos com maior resistência à degradação metabólica, menor toxicidade e actividade gametocitocídica no mínimo idêntica à do fármaco parental [56,57].

As imidazolidin-4-onas 22 são estáveis no plasma humano e em tampão aquoso a pH e temperatura fisiológicos, em claro contraste com os derivados $\mathrm{N}$-acilados seus percursores, e são capazes de prevenir a transmissão de malária berghei entre o mamífero hospedeiro (ratos BalbC) e o mosquito vector (Anopheles stephensi). Esta actividade gametocitocida revelou-se mais pronun- res, que demonstrou ser bem tolerada a nível gastrointestinal.

O tempo de semivida da TAF é de catorze dias, bastante superior às quatro a seis horas da $\mathrm{PQ}$ e comparável ao da mefloquina (duas a três semanas) [59] .Além da eficácia profilática da TAF contra a malária falciparum, relatada neste mesmo estudo [59], surgiram já evidências experimentais da sua eficácia contra outras estirpes do parasita [60].

A TAF também apresenta um certo grau de actividade esquizonticida sanguínea, [61] mas os seus potenciais efeitos adversos, como metemoglobinémia, ainda não estão devidamente esclarecidos. Foi, no entanto, detectada anemia hemolítica em dois casos de administração acidental de TAF, um dos quais através de transfusão sanguínea [2].

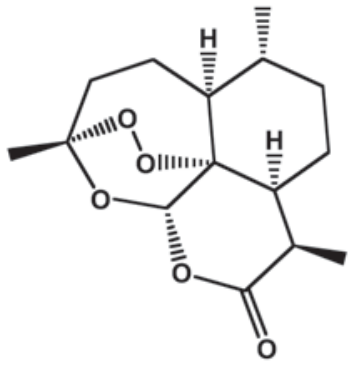

23

Artemisinina

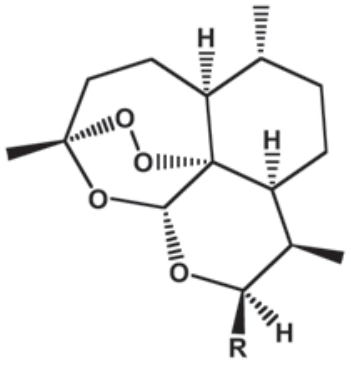

$24(\mathrm{R}=\mathrm{OH})$

Di-hidroartemisinina

$25\left(\mathrm{R}=\mathrm{OCO}\left(\mathrm{CH}_{2}\right)_{2} \mathrm{COOH}\right)$

Artesunato

$26\left(\mathrm{R}=\mathrm{OCH}_{3}\right)$

Artemeter

$27\left(\mathrm{R}=\mathrm{OCH}_{2} \mathrm{CH}_{3}\right)$

Artéter

Figura 9 Estrutura da artemisinina e seus derivados

ciada para os compostos derivados dos aminoácidos glicina e alanina, em detrimento de aminoácidos mais lipofílicos, como a leucina ou a fenilalanina [58]. Estes resultados constituem um exemplo encorajador na busca de fármacos alternativos para o combate à malária.

\section{Tafenoquina}

A tafenoquina (TAF, 19), também designada WR 238 605, é um análogo da PQ já submetido a ensaios clínicos prelimina-
Elubaquina

A elubaquina ( $B Q, 20)$, também designada por bulaquina ou CDRI 80/53, é a mais recente 8 -aminoquinolina que revela propriedades promissoras como agente gametocitocida, sendo derivada da PQ. Os primeiros estudos com este fármaco revelaram que seria cerca de três a quatro vezes menos hepatotóxico do que a $P Q$, apresentando uma extensão de ligação ao citocromo P450 idêntica à desta última [62].
Um trabalho recente, com malária $c y$ nomolgi em macacos rhesus, permitiu comprovar a acção gametocitocida rápida e eficaz da $B Q$, bem como as suas propriedades preventivas contra recidivas da infecção [63]. Estes resultados deram origem a um estudo clínico limitado sobre a actividade da BQ contra malária falciparum em humanos e indica que a $B Q$ é um antimalárico gametocitocida mais eficaz que $P Q$ [63]. No entanto, a BQ é instável nos solventes orgânicos mais comuns e em meio ácido, o que poderá limitar simultaneamente a sua produção e aplicação terapêutica [64].

\section{Fármacos artemisínicos}

A artemisinina (23, Figura 9) é o agente antimalárico de acção mais rápida, originando melhoras significativas do estado febril em apenas 32 horas, em contraste com os dois ou três dias que outros antimaláricos clássicos demoram para provocar efeitos semelhantes. A artemisinina e seus derivados são gametocitocidas, logo, bloqueadores da propagação da doença. No entanto, a sua utilização tem que ser acompanhada por medidas radicais de eliminação total dos mosquitos, dado o curto tempo de semivida que caracteriza esta classe de antimaláricos (cinco a sete dias) [2]. Uma forma alternativa de potenciar a acção dos fármacos artemisínicos reside na sua combinação com outros antimaláricos, como a mefloquina, a lumefantrina ou os antifolatos [14].

O mecanismo de acção dos fármacos artemisínicos tem sido atribuído à quebra da ligação peróxido por reacção do fármaco com o Fe(II) do grupo heme, no vacúolo do parasita, gerando-se radicais que vão alquilar esse grupo heme [2]. Esta hipótese foi apoiada por cálculos teóricos efectuados por Hadipour e colaboradores, que demonstraram que o catião $\mathrm{Fe}^{2+}$ interactua preferencialmente com a ligação peróxido, relativamente aos outros átomos de oxigénio presentes na estrutura do fármaco [65]. No entanto, um trabalho relativamente recente vem refutar esta hipótese, defendendo os seus autores que a artemisinina não é activada pelo ferro hémico no parasita, não sendo necessária a formação de espécies radicalares para que o fármaco 
exerça a sua toxicidade sobre o Plasmodium [66].

Dada a acção rápida da artemisinina, e a aparente incapacidade do parasita em desenvolver resistência contra este fármaco, foram concebidos diversos derivados da artemisinina na busca de melhores perfis terapêuticos. Os derivados artemisínicos mais importantes são a diidroartemisinina (24), o artesunato ou ácido artesúnico (25), o artemeter (26) e o artéter (recentemente designado por artemotil) (27). Os derivados $\mathbf{2 5}$,

26 e 27 têm sido utilizados, em todo o mundo, no tratamento de pacientes com malária severa. Verifica-se que, uma vez activados, destroem rapidamente $\mathrm{o}$ parasita, pelo que são recomendados pela Organização Mundial de Saúde [67].

A di-hidroartemisinina (24) é um metabolito comum aos outros três derivados, exibindo actividade antimalárica igual ou superior à do fármaco parental. Após administração da dose terapêutica, cerca de 50 a $70 \%$ da dihidroartemisina encontra-se ligada às proteínas plasmáticas, em especial, à albumina. A síntese da di-hidroartemisina, a partir da artemisinina, é um processo dispendioso, pelo que muita da informação que existe sobre o fármaco resulta de estudos com artesunato (25) e artemeter (26) [14]. Na realidade, o artesunato é o único derivado hidrossolúvel da artemisinina, sendo tão rapidamente biotransformado em di-hidroartemisinina (24), que pode ser considerado como um pró-fármaco desta [68].

Os derivados artemisínicos têm tido especial interesse na concepção de fórmulas terapêuticas combinadas, envolvendo outros antimaláricos de acção lenta e tempos de semivida muito longos. Esta estratégia visa a redução da duração do tratamento e do desenvolvimento de resistência por parte do parasita. Uma destas formulações terapêuticas combina o artesunato com a mefloquina e é recomendada como terapia padrão na Tailândia [2].
O artemeter e o artéter são derivados lipossolúveis da artemisinina. Após administração oral, o artemeter é rapidamente absorvido no intestino e é extensamente convertido a di-hidroartemisinina no fígado ou no lúmen intestinal [14]. O artéter é um óleo solúvel em éter etílico, que apresenta uma potente actividade esquizonticida sanguínea. Um estudo recente [67], revelou que a velocidade e a extensão da conversão dos isómeros do artéter a di-hidroartemisinina em ratinhos depende da via de administração. Assim, o artéter é rápida e extensamente convertido àquele metabolito após administração oral, esta conversão pode ser substancialmente retardada se a administração for intramuscular. Assim, a via de administração dos derivados artemisínicos poderá ser um elemento-chave na modulação dos efeitos terapêuticos destes antimaláricos [67].

\section{Antifolatos}

Muitos antibióticos clássicos, como as biguanidas ou as sulfonamidas, exer-

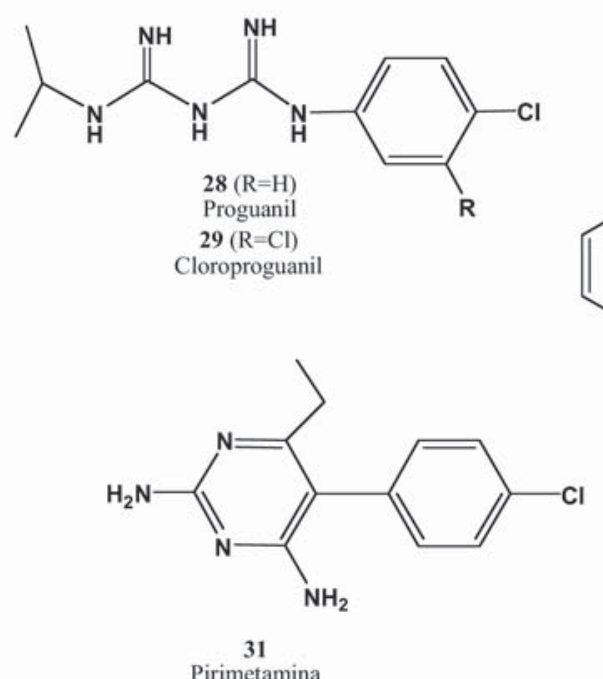<smiles>COc1cc(Cc2cnc(N)nc2N)cc(OC)c1OC</smiles>

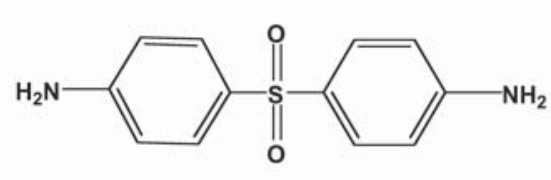

33 Dapsona

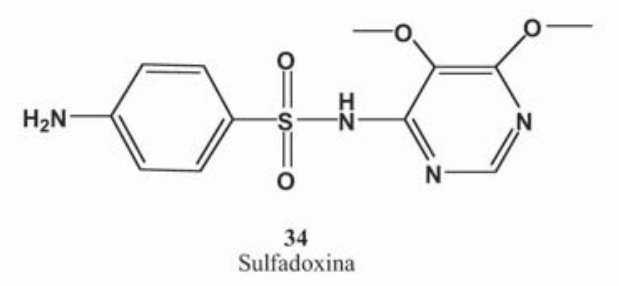

Figura 10 Fármacos antimaláricos que actuam por inibição da biossíntese do folato 
cem a sua acção antibiótica por inibição da biossíntese de folato, um factor de crescimento essencial para o parasita. A biossíntese do folato pode ser comprometida por inibição de duas enzimas, a di-hidropteroato sintetase (DHPS) e a dihidrofolato reductase (DHFR). O metabolismo do folato é um aspecto crítico para a viabilidade do Plasmodium, logo, para o desenvolvimento de fármacos para o tratamento e profilaxia da doença [69]. No entanto, e dada a resistência generalizada dos parasitas face a antibióticos inibidores da biossíntese do folato, os antimaláricos desta família são cada vez menos utilizados com fins terapêuticos. Ainda assim, os antifolatos representam uma classe de antimaláricos importantes, quer por motivos históricos, quer pela sua utilização em terapias combinadas.

Biguanidas

O proguanil (28, Figura 10) e o cloroproguanil (29, Figura 10) são derivados biguanidínicos que actuam como inibidores da DHFR. São considerados pró-fármacos do cicloguanil e clorocicloguanil, respectivamente,[15a] que se formam por via metabólica com intervenção do citocromo P450 [2].

O uso terapêutico do proguanil como monoterápico está limitado pelo desenvolvimento de resistência pelas formas eritrocíticas assexuadas do P. falciparum, mas a sua combinação com a CQ resulta numa fórmula aplicada na profilaxia da doença [14]. Verifica-se, no entanto, que o proguanil oferece melhores resultados quando combinado com a atovaquona (30, Figura 10) [70].

O cloroproguanil apresenta apenas um átomo de cloro adicional relativamente à estrutura do proguanil, e a sua actividade antiplasmódica intrínseca é superior à deste fármaco. Da mesma forma, o seu metabolito clorocicloguanil é mais activo que o correspondente metabolito do proguanil, o cicloguanil [14].

Diaminopirimidinas

As diaminopirimidinas mais usuais no tratamento da malária são a pirimetamina (31, Figura 10) e o trimetoprim (32, Figura 10), geralmente administradas em combinação com sulfonamidas.
Após administração, 90\% da pirimetamina encontra-se ligada às proteínas plasmáticas, sendo metabolizada maioritariamente no fígado [69,71]. O trimetoprim é também um inibidor da DHFR, tendo uma percentagem de ligação às proteínas plasmáticas inferior a 50\%. Em formulações terapêuticas para o tratamento da malária, aparece quase sempre associado ao sulfametaxazole ou outros agentes [14,72].

Dapsona

A dapsona (33, Figura 10) é um inibidor da DHPS com uma actividade antimalárica reduzida. No organismo, é metabolizado a monoacildapsona, que é depois convertido na correspondente hidroxiamina [14]. Apesar da sua baixa actividade, tem vindo a ser usado em associação com outros antimaláricos, como o proguanil ou, mais frequentemente, a pirimetamina, para dar resposta à crescente resistência do parasita às terapias convencionais [73].

\section{Sulfonamidas}

As sulfonamidas são inibidores da DHPS, que encontram utilização como antimaláricos em combinação com outros agentes. O exemplo mais representativo é o da sulfadoxina (34, Figura 10) que, tal como todas as sulfonamidas, se liga extensamente às proteínas plasmáticas [14]. Esta classe de compostos, sem utilidade como monoterapêuticos, tem sido explorada para a obtenção de novos antimaláricos de perfil melhorado [74-76].

\section{Atovaquona}

A atovaquona (30) é uma hidroxi-naftoquinona cuja actividade anti-plasmódica se baseia num mecanismo de acção particular: este fármaco inibe o sistema de transporte de electrões (STE) mitocondrial do parasita, afectando deste modo a biossíntese da pirimidina [77].

A utilização da atovaquona como monoterápico induz mutações no parasita, ao nível do gene do citocromo b localizado no genoma mitocondrial, o que resulta no aumento da resistência à acção do fármaco [78]. Este problema pode ser ultrapassado por combinação da atovaquona com proguanil, numa fórmula terapêutica conhecida como malarone [70].

\section{Considerações finais}

A erradicação da malária é a meta de um caminho já secular e pleno de obstáculos, essencialmente devidos à grande complexidade do ciclo de vida do parasita e à sua versatilidade biológica, que Ihe permite adquirir resistência às inúmeras estratégias quimioterapêuticas que têm vindo a ser aplicadas. A grande panóplia de moléculas activas existente, juntamente com o recente desvelo dos genomas completos de algumas estirpes do parasita e do mosquito transmissor, constituem um estímulo para os esforços dos Químicos, dos Farmacêuticos e dos Médicos no combate à doença $[79,80]$. Sob o ponto de vista médico, moléculas como a dapsona, o cloroproguanil, a pironaridina, a lumefantrina, a artemisinina e seus derivados, ou os novos análogos da cloroquina, estão actualmente a ser submetidas a ensaios clínicos. Paralelamente, os Químicos e os Farmacêuticos estão já a trabalhar na concepção, síntese e caracterização de novas moléculas antimaláricas, em busca não só de maior segurança e eficácia, como também de respostas para novas resistências que provavelmente surgirão. O conhecimento do genoma do parasita abriu uma nova área de estudo e aplicabilidade para futuros compostos-alvos. A mais recente linha de actuação de conceituadas instituições científicas internacionais, votadas à investigação de novos agentes antimaláricos, baseia-se na bioquímica das enzimas e/ou receptores específicos do parasita. Deste modo a concepção de inibidores adequados permitirá afectar o metabolismo normal do parasita em várias frentes, desde a regulação de processos metabólicos essenciais, como a glicólise, à inibição da biossíntese da pirimidina, de ácidos gordos e de proteínas, entre outros [81].

Existe, actualmente, um nível de conhecimento considerável, que poderá contribuir para a erradicação definitiva da malária e de outras doenças tropicais. A crescente mobilidade de pessoas, animais e bens, entre as regiões tropicais do planeta, onde a malária é endémica, e os países ditos desenvolvidos, representa um perigo real de alastramento da doença a estes últimos. Talvez, este 
venha a ser um importante factor de incentivo para o envolvimento do sector privado na luta contra a malária.

\section{Referências}

1 http://rbm.who.int/wmr2005/html/exsummary_en.htm

2 J. Wiesnar, R. Ortmann, H. Jomaa, M. Schlitzer, Angew. Chem. Int. Ed. 42 (2003) 5274.

3 N.J. White, J. Clin. Invest. 113 (2004) 1084.

4 H.P. Rang, M.M. Dale, J.M. Ritter, Farmacologia, Guanabara Koogan, 3. ${ }^{\text {a edi- }}$ ção, 1997.

5 R.G. Ridley, A.T. Hudson, Exp. Opin. Ther. Patents 8 (1998) 121.

6 J. Rengelshausen, J. Burhenne, M. Fröhlich, Y. Tayrouz, S.K. Singh, K.D. Riedel, O. Müller, T. Hoppe-Tichy, W.E. Haefeli, G. Mikus, I. Walter-Sack, Eur. J. Clin. Pharmacol. 60 (2004) 709.

7 W.R.J. Taylor, N.J. White, Drug Safety 27 (2004) 25.

8 S. Krishna, N.J. White, Clin. Pharmacokinet. 30 (1996) 263.

9 J.K. Baird, K.H. Rieckmann, Trends in Paras. 19 (2003) 115.

10 A. Strother, I.M. Fraser, R. Allahyari, B.E. Tilton, Bulletin of the World Organization 59 (1981) 413.

11 K.A. Fletcher, P.F. Barton, J.A. Kelly, Biochem. Pharm. 37 (1988) 2683.

12 Basco et al, Ann. Trop. Med. Paras. 93 (1999) 179.

13 L.J. Bolchoz, R.A. Budinsky, D.C. Mcmillan, D.J. Jollow, J. Pharm. Exp. Ther. 297 (2001) 509.

14 P.T. Giao, P.J. Vries, Clin. Pharmacokinet. 40 (2001) 343.

15 T.M.E. Davis, T.H. Hung, I.K. Sim, H.A. Karunajeewa, K.F. Ilett, Drugs 65 (2005) 75.

15a G. Edwards, P.A. Winstanley, Clin. Pharmacokinet. 27 (1994) 150.

16 C. Gaudebout, E. Pussard, F. Clavier, D. Gueret, J.L. Bras, O. Brandicourt, F. Verdier, Ant. Agent. Chemoth. 37 (1993) 970.

17 F. Ezzet, M.V. Vugt, F. Nosten, S. Looareesuwan, N.J. White, Ant. Agent. Chemoth. 44 (2000) 697.

18 P. Nasveld, S. Kitchener, Trans. Royal Soc. Trop. Med. Hyg. 99 (2005) 2.
19 R. Brueckner, K.C. Lasseter, E.T. Lin, B.G. Schuster, Am. J. Trop. Med. Hyg. 58 (1998) 645

20 N. Valecha, T. Adak, A.K. Bagga, O.P. Asthana, J.S. Srivastava, H. Joshi, V.P. Sharma, Curr. Sci. 80 (2001) 561.

21 R.A. Mirghani, O. Ericsson, J. Cook, P. Yu, L.L. Gustafsson, J. Chromat. B. 754 (2001) 57.

22 D. Brocks, R. Mehvar, Clin. Pharmacokinet. 42 (2003) 1359

23 C.M.S. Menezes, K. Kirchgatter, S.M.D. Santi, G.A. Paula, E.I. Ferreira, Rev. Inst. Med. Trop. S. Paulo 43 (2001) 221.

24 D. Brocks, J.W. Toni, Biopharm. Drug Disp. 20 (1999) 165.

25 J. M. Karle, Antimicrob. Agents Chemother. 41 (1997) 791.

26 D.R. Brocks, Biopharm. Drug Disp. 21 (2000) 365.

27 D.L. Wesche, B.G. Schuster, W.X. Wang, R.L. Woosley, Clin. Pharmac. Therap. 67 (2000) 521

28 P. Winstanley, Br. J. Pharmacol. 42 (1996) 411.

29 T. Matsumura, T. Fujii, T. Miura, T. Koibuchi, T. Endo, H. Nakamura, T. Odawara, A. Iwamoto, T. Nakamura, J. Inf. Chemoth. 11 (2005) 196.

30 G. Lefèvre, M. Bindschedler, F. Ezzet, N. Schaeffer, I. Meyer, M.S. Thomsen, Eur. J. Pharm. Scien. 10 (2000) 141.

31 R. McGready, A. Brockman, T. Cho, D. Cho, M. Vugt, C. Luxemburger, T. Chongsuphajaisiddhi, N.J. White, F. Nosten, Trans. Royal Soc. Trop. Med. Hyg. 94 (2000) 689.

32 F. Grellepois, P. Grellier, D. BonnetDelpon, J.P. Bégué, ChemBioChem. 6 (2005) 648.

33 Y. Khaliq, K. Gallicano, C. Tisdale, G. Carignan, C. Cooper, A. McCarthy, Br. J. Clin. Pharmacol. 51 (2001) 591.

34 M.L. Go, Q.T.Li, Chem. Pharm. Bull. 51 (2003) 241.

35 A. Annerberg, T. Singtoroj, P. Tipmanee, N.J. White, N.P.J. Day, N. Lindegardh, J. Chromat. B. 822 (2005) 330.

36 D. Colussi, C. Parisot, F. Legay, G. Lefèvre, Eur. J. Pharm. Sci. 9 (1999) 9.

37 R. Bakshi, H. Fritz, I. Gathmann, E. Alteri, Trans. Royal Soc. Trop. Med. Hyg. 94 (2000) 419.

38 N. Lindegårdh, A. Annerberg, D. Blessborn, Y. Bergqvist, N. Day, N.J. White, J. Pharm. Biom. Anal. 37 (2005) 1081.
39 P.M. O'Neill, P.G. Bray, S.R. Hawley, S.A. Ward, B.K. Park, Pharmacol. Ther. 77 (1998) 29

40 D. Projean, B. Baune, R. Farinotti, J.P. Flinois, P. Beaune, A.M. Taburet, J. Ducharme, Drug Metab. Disp. 31 (2003) 748.

41 P. Augustijns, N. Verbeke, Clin. Pharmacokinet. 24 (1993) 259.

42 L.M.B. Ursos, P.D. Roepe, Med. Res. Rev. 22 (2002) 465

43 D.J. Naisbitt, J.E. Ruscoe, D. Williams, P.M. O'Neill, M. Pirmohamed, B.K. Park, J. Pharmac. Exp. Ther. 280 (1997) 884

44 F. Lopes, R. Capela, J.O. Gonçalves, P.N. Horton, M.B. Hursthouse, J. Iley, C.M. Casimiro, J. Bom, R. Moreira, Tetrah. Lett. 45 (2005) 7663.

45 T.K. Mutabingwa, R. Hallet, J. Ahmed, C. Drakeley, D. Anthony, A. Heller, Lancet 365 (2005) 1474.

46 S.T. Mariga, J.P. Gil, W.H. Wernsdorfer, A. Björkman, Acta Tropica 93 (2005) 221.

47 S. Gupta, M.M. Thapar, S.T. Mariga, W.H. Wersnsdorfer, A. Björkman, Exp. Parasit. 100 (2002) 28.

48 P. Ringwald, J. Bickii, L. Basco, Lancet 347 (1996) 24

49 G.P. Dutta, S.K. Puri, A. Awasthi, M. Mishra, R. Tripathi, Life Sci. 67 (2000) 759.

50 P. Petmitr, G. Pougvilairat, S. Auparakkitanon, P. Wilairat, Paras. Int. 48 (2000) 275.

51 J. Qi, S. Wang, G. Liu, H. Peng, J. Wang, Z. Zhu, C. Yang, Bioch. Bioph. Res. Comm. 319 (2004) 1124

52 S. Vangapandu, S. Sachdeva, M. Jain, S. Singh, P. Singh, C.L. Kaul, R. Jain, Bioorg. Med. Chem. 11 (2003) 4557.

53 P. Srivastava, S. Singh, G.K. Jain, S.K. Puri, V.C. Pandey, Ecotoxicol Environ. Safety 45 (2000) 236.

54 A.M. Clark, C.D. Hufford, R.K. Puri, J.D. Mcchesney, App. Environ. Microb. 47 (1984) 540.

55 A. Strother, R. Allahyari, J. Buchholz, I.M. Fraser, B.E. Tilton, Drug Metab. Disp. 12 (1984) 35.

56 M.J. Portela, R. Moreira, E. Valente, L. Constantino, J. lley, J. Pinto, R. Rosa, P. Cravo, V. Rosário, Pharm. Res. 16 (1999) 949. 
57 P. Gomes, M.J. Araújo, M. Rodrigues, N. Vale, Z. Azevedo, J. Iley, P. Chambel, J. Morais, R. Moreira, Tetrahedron 60 (2004) 5551.

58 M.J. Araújo, J. Bom, R. Capela, C. Casimiro, P. Chambel, P. Gomes, J. Iley, F. Lopes, J. Morais, R. Moreira, E. Oliveira, V. Rosário, N. Vale, J. Med. Chem. 48 (2005) 888

59 R.P. Brueckner, T. Coster, D.L. Wesche, M. Shmuklarsky, B.G. Schuster, Antimicrob. Agents Chemother. 42 (1998) 1293

60 P. Nasveld, S. Kitchener, Trans. Royal Soc. Trop. Med. Hyg. 99 (2005) 2.

61 J.L. Vennerstrom, E.O. Nuzum, R.E. Miller, A. Dorn, L. Gerena, P.A. Dande, W.Y. Ellis, R.G. Ridley, W.K. Milhous, Antimicrob. Agents Chemother. 43 (1999) 598.

62 P. Srivastava, S.K. Sahni, L.M. Tripathi, S.K. Puri, G.P. Dutta, V.C. Pandey, Bioch. Pharmacol. 43 (1992) 904.

63 S.K. Puri, G.P. Dutta, Exp. Parasit. 111 (2005) 8.
64 J. Lal, N. Mehrotra, R.C. Gupta, J. Pharm. Biomed. Anal. 32 (2003) 141.

65 M. Rafiee, N.L. Hadipour, H. NaderiManesh, J. Chem. Inf. Model. 45 (2005) 366.

66 S. Parapini, N. Basilico, M. Mondani, P. Olliano, D. Taramelli, D. Monti, FEBS Lett. 575 (2004) 91.

67 S. Sabarinath, K.P. Madhusudanan, R.C. Gupta, Biopharm. Drug Disp. 26 (2005) 211.

68 G.A. Balint, Pharm. Ther. 90 (2001) 261.

69 J.E. Hyde, Acta Tropica 94 (2005) 191.

70 M.M. Thapar, J.P. Gil, Bjorkman, Trans. Royal Soc. Trop. Med. Hyg. 99 (2005) 62.

71 A. Agarwal, K. Srivastava, S.K. Puri, M.S. Chauhem, Bioorg. Med. Chem. Lett. 15 (2005) 3133

72 J.E. Renew, C.H. Huang, J. Chromat. A. 1042 (2004) 113

73 S. Krudsood, M. Imwong, P. Wilairatana, S. Pukrittayakamee, A. Nonprasert, G. Snounou, N.J. White, S. Looareesuwan,
Trans. Royal Soc. Trop. Med. Hyg. 99 (2005) 142

74 P. Gomes, J.R.B. Gomes, M. Rodrigues, R. Moreira, Tetrahedron 59 (2003) 7473.

75 J.R.B. Gomes, P. Gomes, Tetrahedron 61 (2005) 2705.

76 J.N. Domínguez, C. León, J. Rodrigues, N.G. Domínguez, J. Gut, P.J. Rosenthal, /l Farmaco 60 (2005) 307

77 D.C. Williams, M.P. Clark, Tetrah. Lett. 39 (1998) 7629.

78 M. Korsinczky, N. Chen, B. Kotecka, A. Saul, K. Rieckmann, Q. Cheng, Antim. Agents Chemoth. 44 (2000) 2100.

79 G.A. Biagini, P.M. O'neill, P.G. Bray, S.A. Ward, Curr. Opin. Pharm. 5 (2005) 1.

80 http://www.nature.com/nature/malaria/ index.html

81 R. Pink, A. Hudson, M.A. Mouriès, M. Bendig, Nature 4 (2005) 727.

\section{Limpeza com Ureia}

Em 1 de Outubro de 2005, os limites para as emissões máximas de óxidos de azoto na União Europeia foram reduzidos em $30 \%$ para os camiões e $50 \%$ para os carros movidos a motor diesel. A partir de 2008, os regulamentos tornar-se-ão ainda mais apertados quer na Europa, quer nos EUA.

As emissões de óxidos de azoto dos camiões representam $40 \%$ do total das emissões destes poluentes provenientes do tráfego automóvel e, desse modo, suscitam uma importante preocupação ambiental. Conversores catalíticos de três vias reduzem consideravelmente as emissões provenientes de veículos movidos a gasolina, mas a sua aplicação ao diesel ainda se revela problemática até ao momento. No entanto, estas preocupações podem fazer parte do passado, já que, investigadores da Universidade de Lund demonstraram que a injec- ção de ureia na corrente dos gases de exaustão, induz uma reacção de redução dos óxidos de azoto. O investigador Ingemar Odenbrand afirma que deste modo a ureia é convertida em amoníaco, reduzindo o óxido de azoto ao inócuo azoto molecular. Por outro lado, também contribui para a redução da quantidade de partículas residuais, igualmente prejudiciais.

A equipa de Lund tem trabalhado com a Volvo, a Scania, o fabricante de conversores catalíticos Johnson Mattey e o Instituto de Tecnologia Chalmers, em Gotemburgo de forma a desenvolver um método de armazenamento de óxidos de azoto, $\mathrm{NO}_{\mathrm{x}}$, que possibilite uma redução ainda maior das emissões. Este método é baseado na retenção do $\mathrm{NO}_{x}$ no conversor catalítico que é sucessivamente reduzido através da repetida injecção de hidrocarbonetos (normalmente, combustível diesel) em intervalos de tempo de 60 ou 90 segundos. Os hidrocarbone- tos são partidos em hidrocarbonetos mais pequenos e monóxido de carbono, que reduzem o $\mathrm{NO}_{x}$ a azoto molecular. A equipa de investigação refere que as suas experiências possibilitaram uma redução das emissões de 7 $\mathrm{g}$ de $\mathrm{NO}_{\mathrm{x}}$ por $\mathrm{kWh}$ a $3 \mathrm{~g}$.

Usando um sistema de motor e exaustão à escala real, os investigadores estão a aperfeiçoar o processo de forma a levar em conta as variações de temperatura, caudal e consistência que ocorrem em correntes de exaustão reais. O objectivo consiste em verificar as especificações requeridas pela legislação ambiental dos EUA, que deverão entrar em vigor em 2008, e que implicarão uma redução de $94 \%$ dos níveis de emissão de $\mathrm{NO}_{x}$ em relação aos valores actuais. (adaptado de webzine Reactive Reports (2005) 48).

Paulo Brito 